\title{
Low-dose ionizing irradiation triggers apoptosis of undifferentiated spermatogonia in vivo and in vitro
}

\author{
Lixin $\mathrm{Qi}^{1}$, Jiaxuan $\mathrm{Li}^{1}$, Wei Le ${ }^{1}$, Jinfu Zhang ${ }^{1,2}$ \\ ${ }^{1}$ Department of Urology, Tongji Hospital, Tongji University School of Medicine, Shanghai 200065, China; ${ }^{2}$ Department of Urology, Tongren \\ Hospital, Shanghai Jiao Tong University School of Medicine, Shanghai 200050, China \\ Contributions: (I) Conception and design: J Zhang, W Le; (II) Administrative support: W Le; (III) Provision of study materials or patients: All authors; \\ (IV) Collection and assembly of data: L Qi, J Li; (V) Data analysis and interpretation: L Qi; (VI) Manuscript writing: All authors; (VII) Final approval \\ of manuscript: All authors. \\ Correspondence to: Jinfu Zhang, MD, PhD. Department of Urology, Tongren Hospital, Shanghai Jiao Tong University School of Medicine, 1111 \\ Xianxia Road, Shanghai 200050, China. Email: zhangjinfu@tongji.edu.cn; Wei Le, MD. Department of Urology, Tongji Hospital, Tongji University \\ School of Medicine, 389 Xincun Road, Shanghai 200065, China. Email: 2016lewei@tongji.edu.cn.
}

Background: The present study aimed to investigate the mechanism of low-dose ionizing radiation (IR) induced apoptosis of undifferentiated spermatogonia in vivo and in vitro.

Methods: Following $50 \mathrm{mGy}$ IR, testicular tissues were collected from the adult DBA/2 mice at 1, 2 and $24 \mathrm{~h}$; mice in the control group received pseudo-irradiation. Immunofluorescence (IF) staining and TUNEL were performed to assess DNA damage and apoptosis, respectively, in the irradiated testicular tissues. Furthermore, the spermatogonia were also irradiated in vitro, and the expression of apoptosis-related proteins was detected by Western blotting. TUNEL and flow cytometry were applied to assess cell apoptosis.

Results: $\gamma \mathrm{H} 2 \mathrm{AX}$ (a marker of DNA damage) was up-regulated in the seminiferous tubules at 1 and $2 \mathrm{~h}$ after IR, but it was reduced following the DNA repair. This was consistent with the finding that apoptosis of germline cells was present in the seminiferous tubules after IR, especially at $1 \mathrm{~h}$ (IF and TUNEL). Apoptosis was also present in the $\operatorname{PLZF}(+)$ spermatogonia, particularly at $1 \mathrm{~h}$ after IR. Apoptotic cells decreased with the increase in DNA repair time after IR. Moreover, the caspase-3 protein was expressed in the undifferentiated spermatogonia following IR. The expression of caspase-3, P53, Ku70 and DNA-PKcs in the cultured spermatogonia was also up-regulated following IR in vitro, but their expression decreased gradually over time after IR, which was supported by the findings from flow cytometry, and the apoptosis of spermatogonia peaked at $24 \mathrm{~h}$ post IR.

Conclusions: IR may induce the apoptosis of spermatogonia at early stage in vivo, but the apoptosis of spermatogonia secondary to IR occurs at a relatively later time point $(24 \mathrm{~h})$ in vitro mainly. The apoptosis of spermatogonia is improved over time after IR.

Keywords: Undifferentiated spermatogonia; apoptosis; irradiation

Submitted Aug 18, 2019. Accepted for publication Sep 29, 2019.

doi: $10.21037 /$ tau.2019.10.16

View this article at: http://dx.doi.org/10.21037/tau.2019.10.16

\section{Introduction}

DNA damage is widely observed in the eukaryotic cells. They are two types of DNA damage: single strand break (SSB) and double strands break (DSB). Many factors contribute to DNA DSB, including reactive oxygen species (ROS), formation of replication forks during cell replication and ionizing irradiation (IR). IR causes cell genomic DNA DSBs, which directly affects cell genomic stability. DSBs have been reported to trigger the most detrimental effects on the genome stability and identified as the main contributor to IR-induced cell-killing effect through the formation of chromosomal aberrations. Studies 
have shown that IR may adversely affect male germ cells, and further influences male spermatogenesis $(1,2)$. The male testicular germ cells are more sensitive to DNA DSB than other tissues (3). Radiotherapy, chemotherapy and other treatments for tumors may also cause DNA damage of germ cells, resulting in infertility $(4,5)$. Undifferentiated spermatogonia (As, Apr and Align) is a unique spermatogonia in adult male testes and plays an important role in maintaining fertility and spermatogenesis. Although it has been reported that low-dose IR may cause DNA DSB in the spermatogonia, the relationship between low-dose IR and apoptosis of undifferentiated spermatogonia/progenitor cells is still poorly understood. Therefore, the present study aimed to investigate the low-dose IR induced DNA DSB and explore the relationship between the DNA DSB and the apoptosis in undifferentiated spermatogonia in vivo and in vitro.

\section{Methods}

\section{Establishment of germline cell DNA damage mouse model}

This study was approved by the Ethics Committee of Tongji Hospital. Twelve one-month-old DBA/2 male mice were divided into four groups, according to DNA damagerepair time (1, 2, $24 \mathrm{~h}$ and control). The total dose of irradiation (Mark I-68A, JL Shepherd and Associates, San Fernando, CA, USA; irradiation condition: $0.1 \mathrm{~Gy} / \mathrm{min}$ for $30 \mathrm{~s}$ ) was applied in the radiation groups, and in the control group, mice were received pseudo-irradiation. The testes were collected at different time points of DNA repair (1, $2,24 \mathrm{~h}$ ) after IR. The testicular tissues were fixed in $4 \%$ paraformaldehyde and then sectioned (10 $\mu \mathrm{m}$ in thickness).

\section{Immunofluorescence staining of testicular sections}

The testicular tissue sections were blocked in a solution containing $2 \%$ BSA, $0.05 \%$ Tween 20 and $0.1 \%$ Triton $\mathrm{X}-100$ at $4{ }^{\circ} \mathrm{C}$ overnight, and then incubated with mouse anti- $\gamma \mathrm{H} 2 \mathrm{AX}$ (Millipore, 05-636, Burlington, USA) at 1:200, rabbit anti-promyelocytic leukemia zinc finger (PLZF; Santa Cruz, sc-22839, Dallas, USA) at 1:200, anti-caspase (Abcam, ab13847, Cambridge, MA, USA) at $4{ }^{\circ} \mathrm{C}$ overnight. Subsequently, the sections were incubated with secondary antibodies at $1: 200$ overnight at $4{ }^{\circ} \mathrm{C}$. The secondary antibodies were rabbit anti-mouse IgG $(\mathrm{H}+\mathrm{L})$ superclonal antibody, Alexa Fluor 555 (Invitrogen, A27028, Waltham, USA), goat anti-rabbit IgG $(\mathrm{H}+\mathrm{L})$ highly cross-absorbed antibody, Alexa Fluor 488 (Invitrogen, A-11034, Waltham, USA). Cell nuclei were counter-stained with $0.3 \%$ DAPI (Beyotime Biotechnology, Shanghai, China). Sections were rinsed with PBS containing $0.05 \%$ Tween 20 and then mounted with anti-fade mounting medium.

\section{TUNEL staining of germ cells in testicular tissue sections}

Proteinase $\mathrm{K}$ working solution was diluted with PBS at a final concentration of $20 \mu \mathrm{g} / \mathrm{mL}$. Each section was treated with $100 \mu \mathrm{L}$ of proteinase $\mathrm{K}$ working solution at room temperature (RT) for $10 \mathrm{~min}$. After rinsing with PBS for 2-3 times, each section was incubated with $100 \mu \mathrm{L}$ of $1 \times$ equilibration buffer for 10-30 min at RT, followed by addition of a solution containing $50 \mu \mathrm{L}$ of TdT enzyme. Then, the sections were incubated at $37^{\circ} \mathrm{C}$ for $60 \mathrm{~min}$, followed by rinsing with PBS containing $0.1 \%$ Triton X-100 and $0.05 \%$ Tween 20. Subsequently, anti-PLZF (Santa Cruz, sc-22839, Dallas, USA) antibody at 1:200 was added to each sample, followed by incubation at $4{ }^{\circ} \mathrm{C}$ overnight. On the next day, the sections were rinsed with PBS containing $0.1 \%$ Triton X-100 and $0.05 \%$ Tween 20 , and then incubated with secondary antibody at RT for $2 \mathrm{~h}$. The nuclei were counterstained with $0.3 \%$ DAPI solution.

\section{Culture and proliferation of mouse spermatogonia}

The primary germline stem cells were collected and cultured with the procedures reported in our previous study (6). In brief, the testes were collected from 4-week-old male DBA/2 mice and then treated with Collagenase type IV at $1 \mathrm{mg} / \mathrm{ml}$ (Sigma, C5138, St. Louis, USA) and $0.25 \%$ Trypsin (Gibco, 25200072, Waltham, USA) to release the testicular cells. THY-1 (CD90) positive germ cells were sorted with the magnetic bead sorting system (MACS). The sorted cells were transferred to CF1 MEF (mouse embryonic fibroblast) feeder cells for further culture. The undifferentiated spermatogonia cells were cultured in the medium containing StemPro-34 (Life Technologies, Carlsbad, CA, USA, 10639-011), StemPro-34 supplement at $20 \mu \mathrm{L} / \mathrm{mL}$ (Life Technologies, 10639-011), insulin at $25 \mu \mathrm{g} / \mathrm{mL}$ (Sigma, 13536), transferrin at $100 \mu \mathrm{g} / \mathrm{mL}$ (Sigma, T1147), pyruvic acid at $100 \mu \mathrm{g} / \mathrm{mL}$ (Sigma, P5280), putrescine at $60 \mu \mathrm{M}$ (Sigma, P5780), sodium selenite at $30 \mathrm{nM}$ (Sigma, S5261), D-(+)-glucose at $6 \mathrm{mg} / \mathrm{mL}$ (Sigma, 49139), lactic acid at $1 \mu \mathrm{L} / \mathrm{mL}$ (Sigma, L4263), L-glutamine at $2 \mathrm{mM}$ (Life Technologies, 25030-081), 2-mercaptoethanol $5 \times 10^{-5}$ (Merck Millipore, Darmstadt, 
Germany, ES-007-E), minimal essential medium (MEM) vitamin solution 19 (Life technologies, 11120-052), MEM non-essential amino acid solution 19 (Life Technologies, 11120-052), 10-4 M ascorbic acid (Sigma, A4403), d-Biotin at $10 \mu \mathrm{g} / \mathrm{mL}$ (Sigma, 14400), b-estradiol at $30 \mathrm{ng} / \mathrm{mL}$ (Sigma, E2758), progesterone at $60 \mathrm{ng} / \mathrm{mL}$ (Sigma, P5096), human basic fibroblast growth factor at $10 \mathrm{ng} / \mathrm{mL}$ (Life Technologies, 13256-029), murine leukemia inhibitory factor at $103 \mathrm{U} / \mathrm{mL}$ (Merck Millipore, Temecula, CA, USA, ESG1107), recombinant human glial cell linederived neurotrophic factor (GDNF) at $10 \mathrm{ng} / \mathrm{mL}$ (Life Technologies, PHC7041), and 1\% fetal calf serum (FCS; Life Technologies, 10099- 141).

\section{Western blotting for detecting apoptosis-related proteins in undifferentiated spermatogonia}

Spermatogonia cells were collected at different time points after IR. The cells were lysed with RIPA lysis buffer containing $1 \mathrm{mM}$ proteinase inhibitor PMSF. BCA protein assay kit (Beyotime Biotechnology, Shanghai, China) was used to determine the protein concentration. Protein samples were separated by 10-12\% SDS-PAGE and then transferred onto 0.45 - $\mu \mathrm{m}$ PVDF membrane (Millipore, Burlington, USA), which was then incubated with specific antibodies: P53 (Abcam, PAb 240), p-P53 (Ab1431), Ku70 (Santa Cruz, sc-365766), DNA-PKcs (Ab32566), caspase-3 and GAPDH (SC-32233).

\section{Detection of apoptosis in undifferentiated spermatogonia by flow cytometry}

The cultured germline cells were irradiated at $50 \mathrm{mGy}$, and then collected at pre-designed time points. To remove MEF cells, the cell suspension was digested with $0.05 \%$ trypsin solution and then transferred to $0.2 \%$ gelatin coated dish, followed by incubation for $2 \mathrm{~h}$. The floating cells were collected and rinsed with PBS. Annexin V (BD) and propidium iodide (PI) were added for staining. After 15-min incubation, cells were subjected to flow cytometry.

\section{Results}

Low-dose IR induced DNA DSB in germ cells of testicular seminiferous tubules

IF staining indicated the expression of $\gamma \mathrm{H} 2 \mathrm{AX}$ (a marker of DNA DSB) in the germ cells of mouse testicular seminiferous tubules after IR (Figure 1). $\gamma \mathrm{H} 2 \mathrm{AX}$ was highly expressed in the seminiferous tubules, especially at 1 and 2 $\mathrm{h}$ after IR, and its expression decreased over time after IR, suggesting that the DNA damage is repaired by germline cells (Figure 2).

\section{Low-dose IR triggered germline cells apoptosis in seminiferous tubules of mouse testes}

TUNEL showed TUNEL positive germline cells in the testicular seminiferous tubules after IR (Figure 3). Apoptotic germline cells increased significantly after IR as compared to the control group $(\mathrm{P}<0.05)$. The apoptosis of germ cells peaked at $24 \mathrm{~h}$ after IR (Figure 4 ).

\section{Low-dose IR induced DNA damage triggered apoptosis of undifferentiated spermatogonia in testicular seminiferous tubules}

PLZF double staining TUNEL was employed to investigate the apoptosis of PLZF(+) undifferentiated spermatogonia in which the progenitor spermatogonia was positive to PLZF. Results showed the apoptotic undifferentiated spermatogonia were also found in the testicular seminiferous tubules after IR. Moreover, the apoptosis of PLZF(+) spermatogonia peaked at $1 \mathrm{~h}$ after IR (Figures 3,5). Similarly, the caspase-3 expression was also observed in the PLZF(+) cells after IR, even at 24 h after IR (Figure 6).

\section{Apoptosis-related proteins expression increased in undifferentiated spermatogonia in vitro post IR}

The expression of Ku70 (a marker of DNA damage) and DNA-PKcs was up-regulated in the undifferentiated spermatogonia after IR, suggested that low-dose IR also induces DNA damage in the progenitor spermatogonia in vitro. The expression of apoptosis-related proteins P53 and p-P53 was also up-regulated in the undifferentiated spermatogonia. Caspase-3 expression increased consistently after IR, and the cleaved caspase-3 expression also increased significantly as compared to the control group after IR $(\mathrm{P}<0.05)$ (Figure 7).

\section{Apoptotic undifferentiated spermatogonia increased in vitro after IR}

Flow cytometry was done to determine the apoptotic undifferentiated spermatogonia after IR. Results showed 


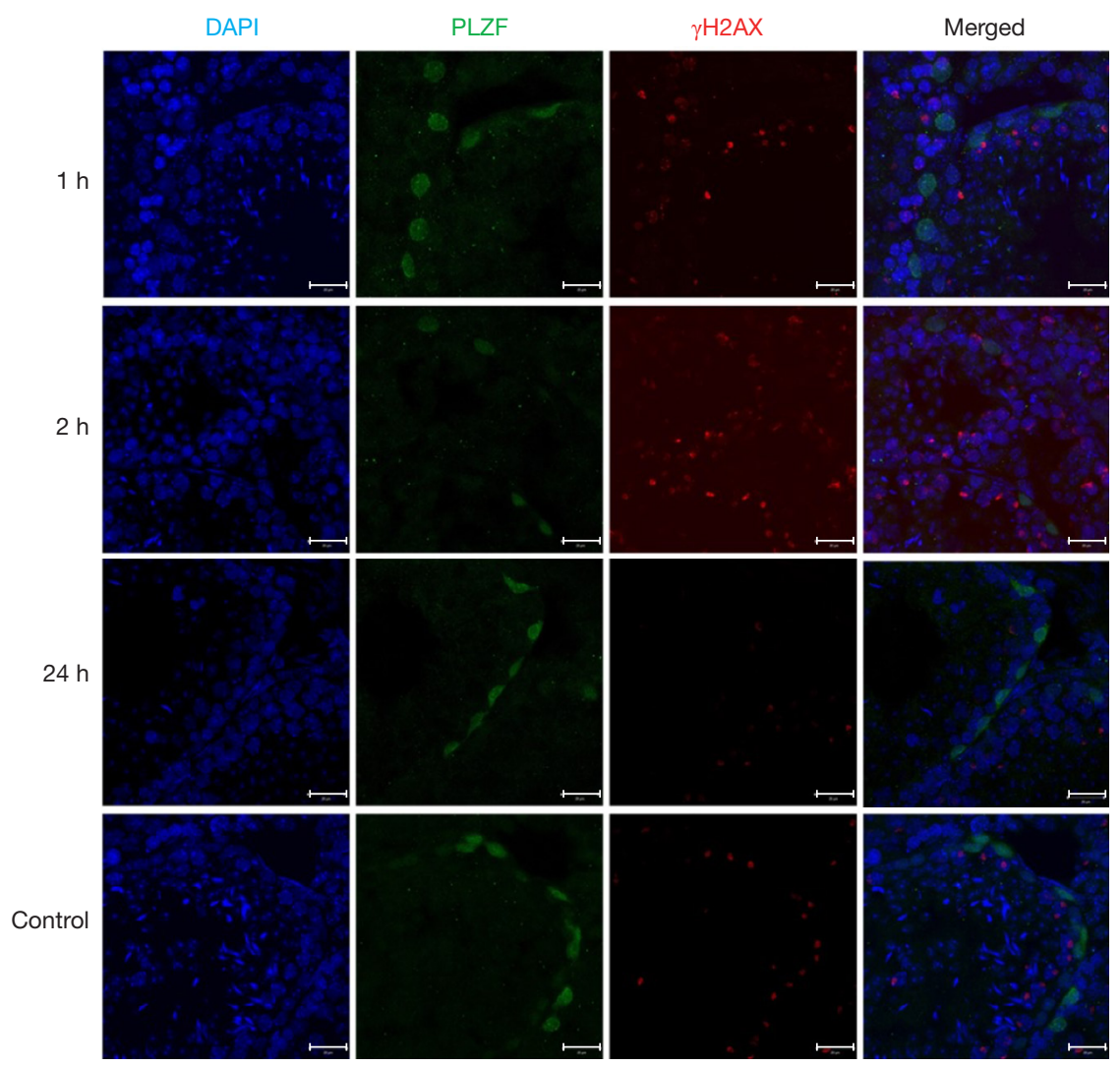

Figure $1 \gamma \mathrm{H} 2 \mathrm{AX}$ expression in the seminiferous tubules after low-dose IR. Testicular tissues were collected at different time points (1, 2 and $24 \mathrm{~h}) . \gamma \mathrm{H} 2 \mathrm{AX}$ expression was observed in the tissues treated with IR; the high intensity $\gamma \mathrm{H} 2 \mathrm{AX}$ signals represented the germline cells at meiosis stage. $\gamma \mathrm{H} 2 \mathrm{AX}$ signals were up-regulated at 1 and $2 \mathrm{~h}$ after IR. PLZF positive spermatogonias were mainly found in the basal region of the seminiferous tubules. Scale bar, $25 \mu \mathrm{m}$.

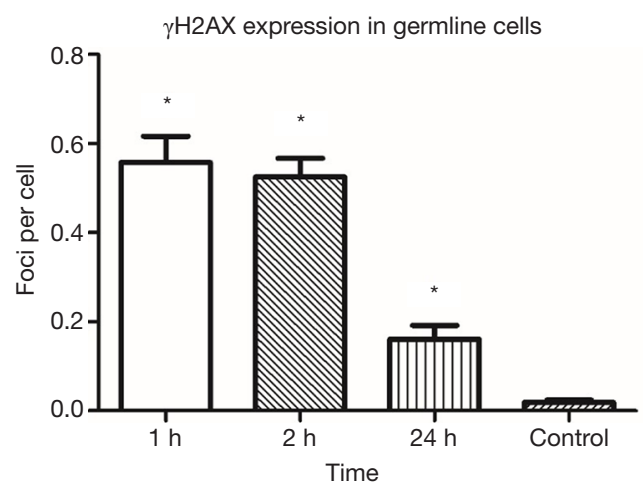

Figure $2 \gamma \mathrm{H} 2 \mathrm{AX}$ expression in the germline cells after IR in vivo. The $\gamma \mathrm{H} 2 \mathrm{AX}$ expression was statistically analyzed. $\gamma \mathrm{H} 2 \mathrm{AX}$ expression was significantly unregulated as compared to the control group. $\gamma \mathrm{H} 2 \mathrm{AX}$ expression decreased over time after IR, suggesting that the DNA DSBs were repaired in the germline cells. More than 10 sections were examined, and more than 200 cells were counted ${ }^{*}, \mathrm{P}<0.05$ vs. control group). IR, ionizing irradiation; DSB, double strands break. that apoptosis peaked at a relatively later time point $(24 \mathrm{~h})$. Apoptotic cells dramatically increased after IR as compared to the control group, including the early stage (annexin $\mathrm{V}$ positive, PI negative) and late stage (annexin $\mathrm{V}$ positive, PI positive) apoptotic undifferentiated spermatogonia $(\mathrm{P}<0.05)$ (Figures 8,9).

\section{Discussion}

DNA damage is common in the eukaryotic cells. The most serious effect of IR on the eukaryotic cells may be DNA DSB and genomic instability $(7,8)$. Many cancer patients usually receive radiotherapy, and the dose of radiation is often higher than that used in animal studies (9). Currently, radiological examination and treatments are widely used in clinical diagnosis and treatment. The precise mechanism of biological effects of irradiation on the undifferentiated 


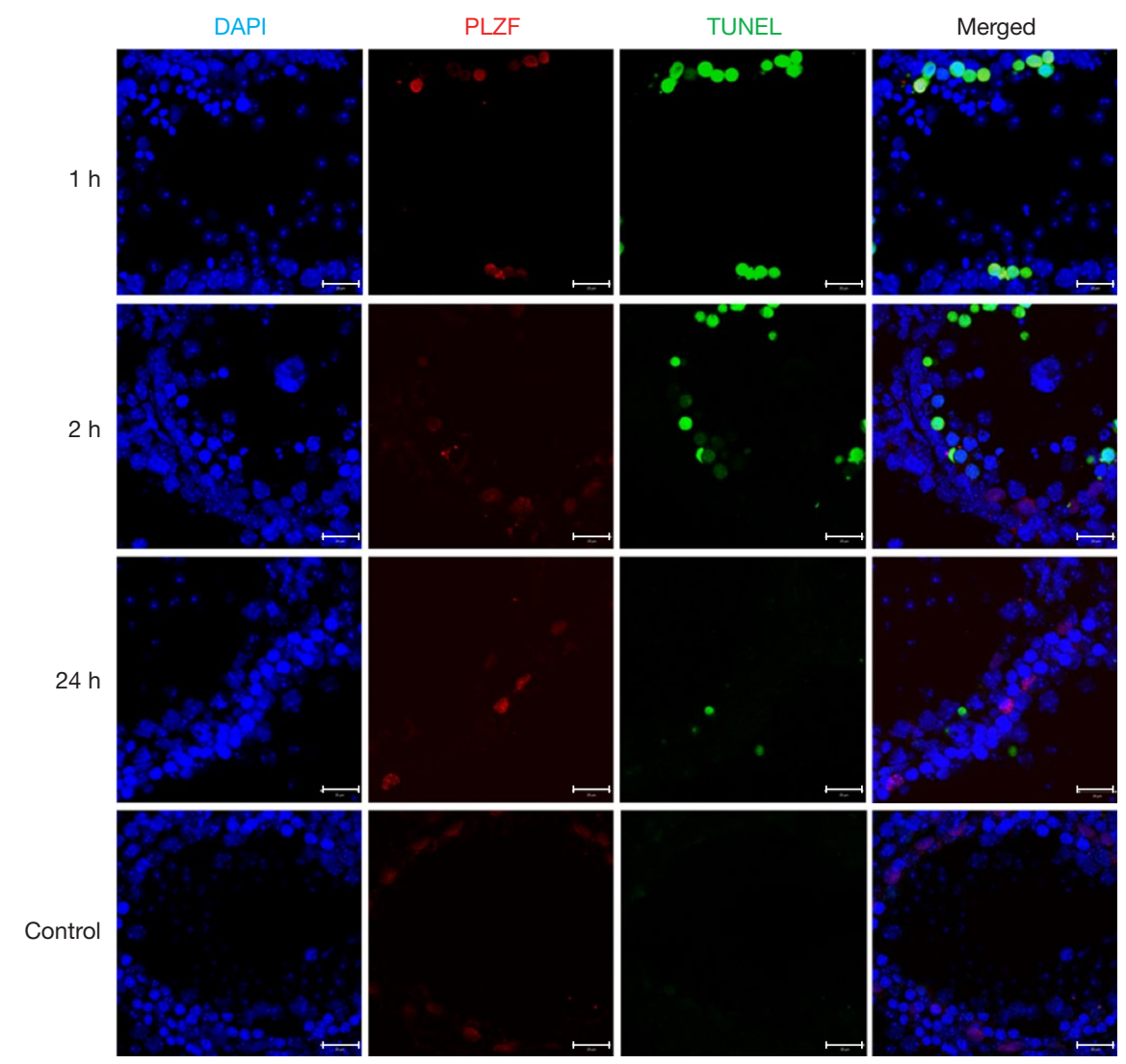

Figure 3 Apoptotic cells in the seminiferous tubules after IR. TUNEL staining was applied to detect the apoptosis of germline cells after IR. TUNEL staining showed the apoptotic germline cells increased significantly after IR and TUNEL positive signals were still observed at $24 \mathrm{~h}$. Some TUNEL positive cells were also found in PLZF(+) spermatogonia, and the apoptosis of germline progenitor cells peaked at 1 and $2 \mathrm{~h}$ after IR. IR, ionizing irradiation. Scale bar, $25 \mu \mathrm{m}$. IR, ionizing irradiation.

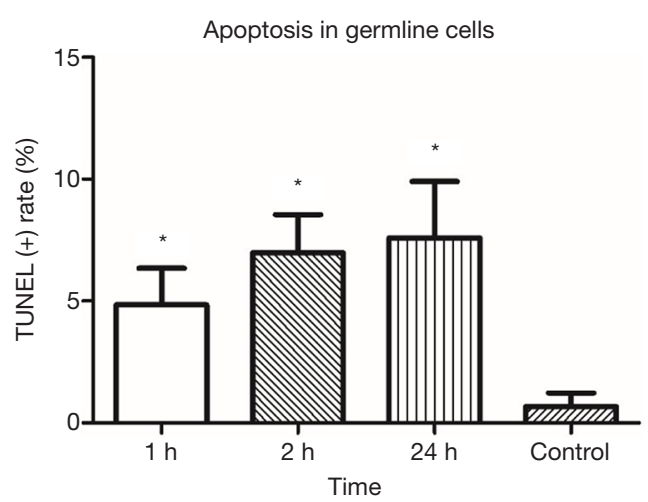

Figure 4 Apoptosis of germline cells after IR. TUNEL positive cells were analyzed in different groups. After IR, the apoptosis rates of germline cells increased as compared to the control group. More than 10 sections were examined, and more than 200 cells were counted $\left({ }^{*}, \mathrm{P}<0.05\right.$ as control group). IR, ionizing irradiation.

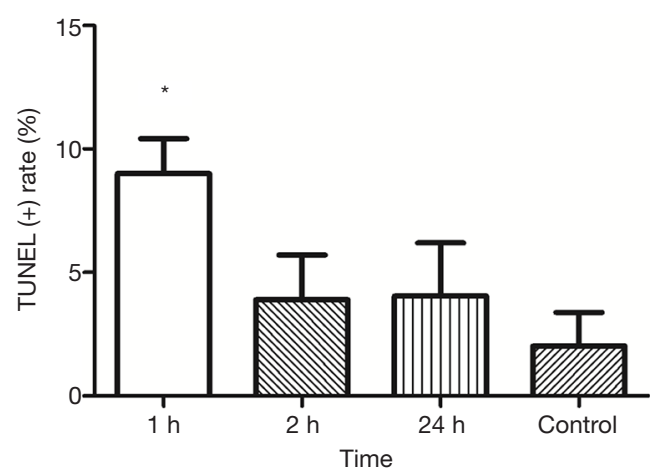

Figure 5 Apoptosis of undifferentiated spermatogonia after IR. TUNEL positive cells were counted in PLZF positive spermatogonias. The apoptosis of undifferentiated spermatogonia peaked at $1 \mathrm{~h}$ after IR. TUNEL positive cells were still observed at 2 and $24 \mathrm{~h}$, however, the number of TUNEL positive cells was similar to that in the control group. More than 10 sections were examined, and more than 200 cells were counted (*, $\mathrm{P}<0.05$ vs. control group). IR, ionizing irradiation. 


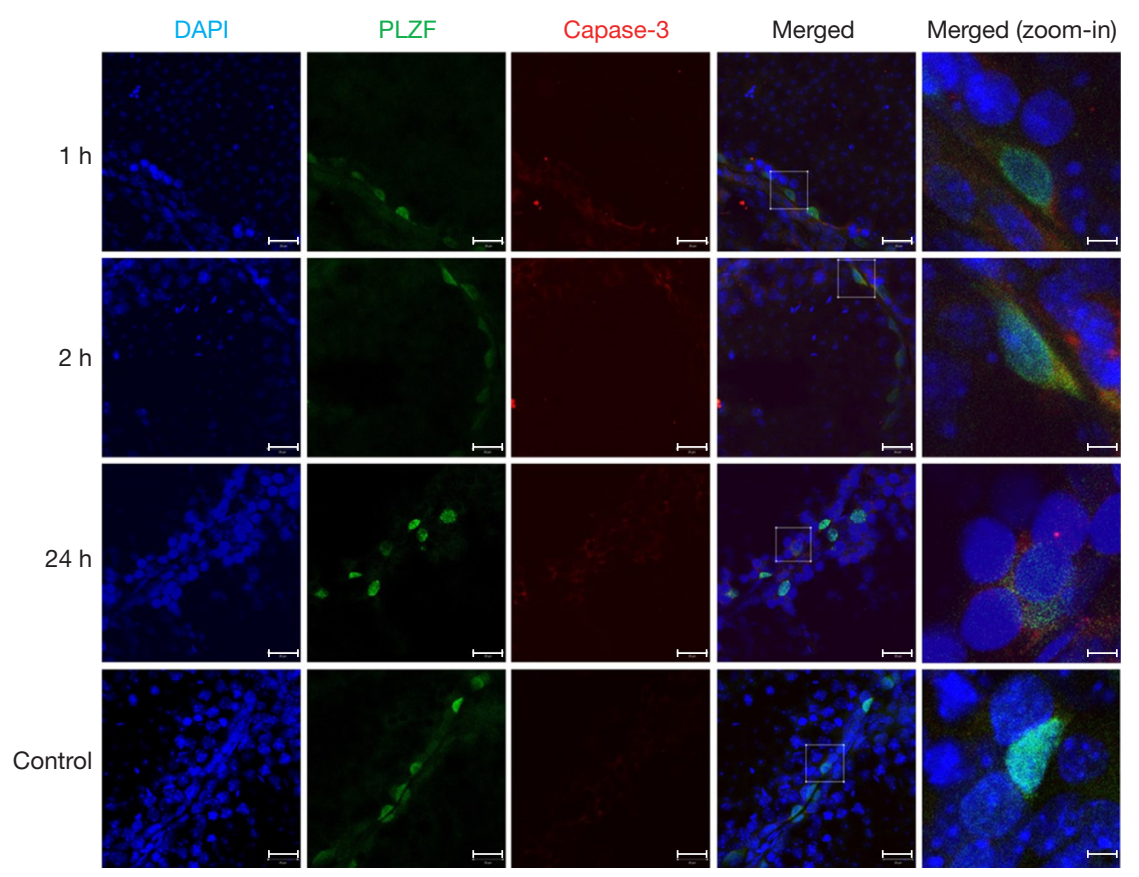

Figure 6 Caspase-3 expression in the undifferentiated spermatogonia after IR. IF staining was performed to detect the apoptosis-related protein (caspase-3) expression in PLZF(+) spermatogonias after IR. Caspase-3 expression was observed in PLZF(+) cells, mainly in the membrane of seminiferous tubules, after IR, while caspase-3 expression was not observed in the control group. Caspase-3 expression was still observed at $24 \mathrm{~h}$ after IR. Scale bar, $25 \mu \mathrm{m}$. IR, ionizing irradiation; IF, immunofluorescence.

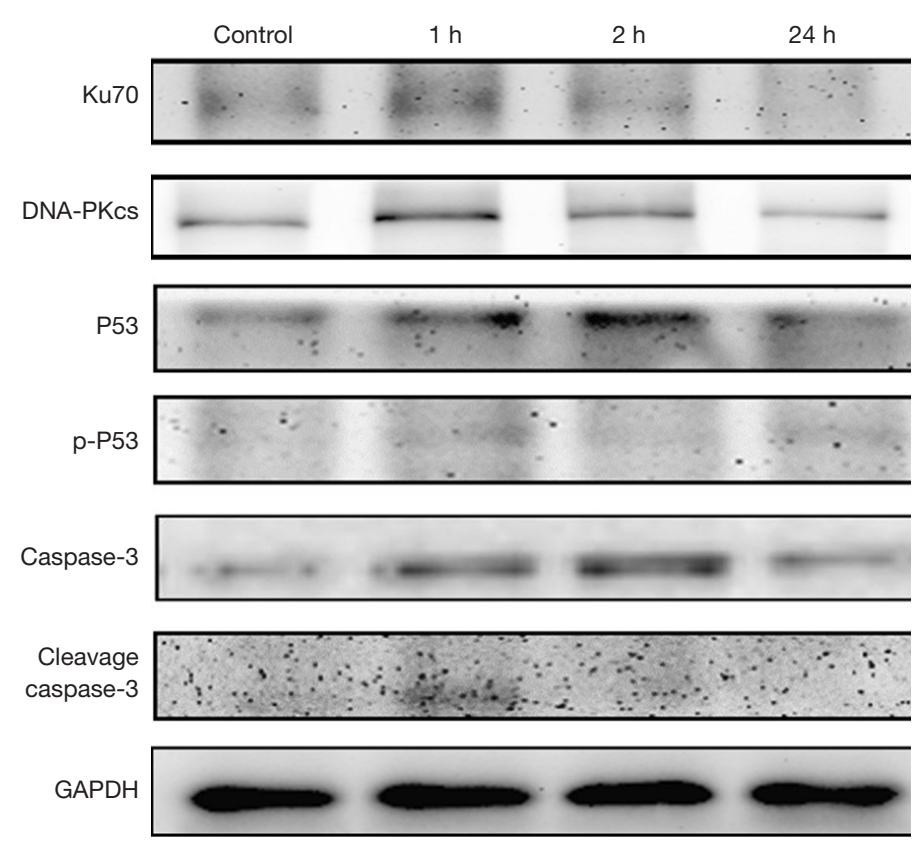

Figure 7 Apoptosis related protein expression in undifferentiated spermatogonia after IR. The expression of DNA DSBs related protein, Ku70 and DNA-PKcs, was up-regulated after IR. Similarly, the expression of apoptosis-related proteins P53, phosphorylated P53 (S15) and caspase-3 was up-regulated in the undifferentiated spermatogonias at different time points (1, 2 and $24 \mathrm{~h})$ after IR. The expression of cleaved caspase-3, active form of caspase-3, was up-regulated at $1 \mathrm{~h}$ after IR. IR, ionizing irradiation; DSB, double strands break. 

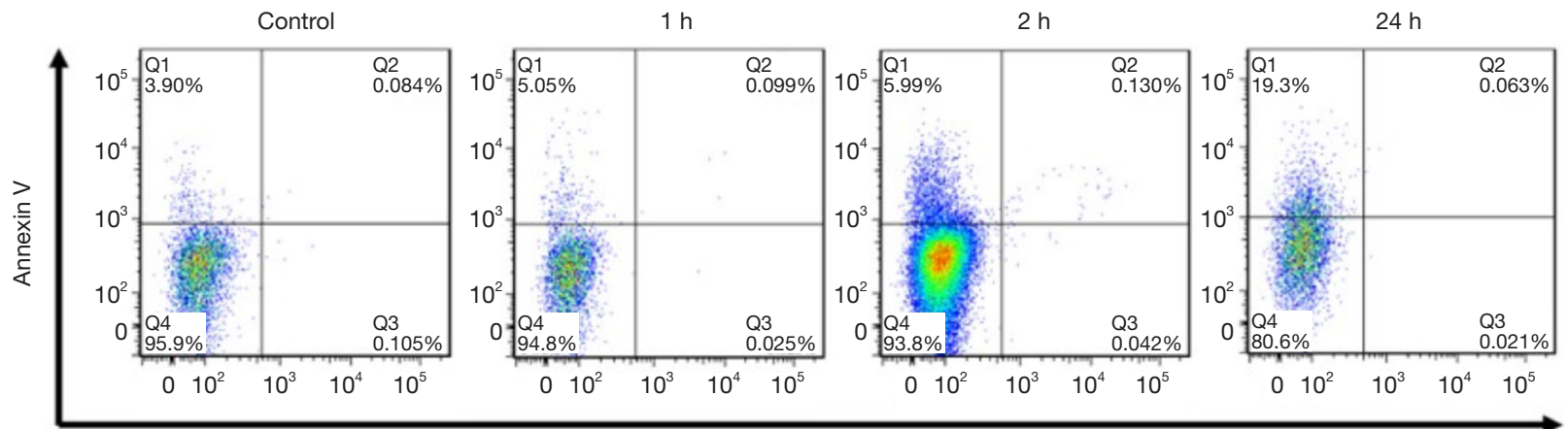

PI

Figure 8 Flow cytometry of undifferentiated spermatogonia after low dose IR. After irradiation, cells were stained with annexin V and PI, followed by flow cytometry for the detection of apoptosis. Apoptosis of undifferentiated spermatogonias was classified as early-stage (annexin $\mathrm{V}$ positive, PI negative) and late-stage (annexin V positive, PI positive) one. IR, ionizing irradiation; PI, propidium iodide.

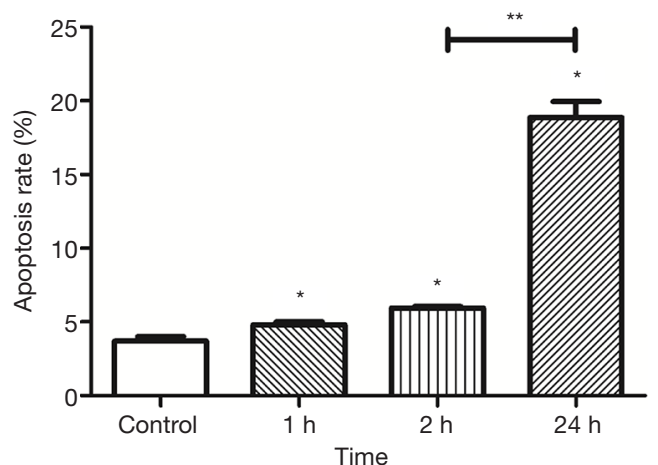

Figure 9 In vitro apoptosis of undifferentiated spermatogonia after IR. Apoptosis of spermatogonia was analyzed by flow cytometry. Apoptosis rate increased after IR as compare to control group. The apoptosis of spermatogonia was more obvious in $24 \mathrm{~h}$ group as compared to other two IR groups. ( ${ }^{*}, \mathrm{P}<0.05$ vs. control group; **, $\mathrm{P}<0.05$ vs. $2 \mathrm{~h}$ group). IR, ionizing irradiation.

germline cells is still unclear, although studies have shown that DNA damage can affect spermatogenesis and is related to male infertility $(5,10)$.

In the present study, THY-1 was used as a MACS marker to sort the undifferentiated spermatogonia. It has been reported that there is only a small proportion of undifferentiated germline cells in the adult mice testes (11). THY-1 is a specific surface marker specifically expressed on As, Apr and Align spermatogonia, and it has been recognized as a marker commonly used for fluorescenceactivated cell sorting (FACS) or MACS sorting of undifferentiated spermatogonia (12). In some studies, THY1 is used as a cell surface marker for sorting undifferentiated spermatogonia $(13,14)$. Although not all the undifferentiated spermatogonias are THY-1 positive, THY-1 is still an ideal indicator of progenitor spermatogonia $(12,15)$. In the present study, undifferentiated spermatogonias were successfully isolated and purified in vitro by using THY-1 as a surface marker as previously reported $(14,16)$.

PLZF is expressed in the nucleus of progenitor germline cells and has been recognized as another marker of undifferentiated spermatogonia (17). PLZF is indispensable for undifferentiated spermatogonia (especially spermatogonial stem cells) in maintaining stemness and self-renewal. PLZF deficiency may cause progressive loss of germline cells, leading to infertility in mammalians (18). Previous studies have demonstrated that PLZF expression gradually decreases during the process of Align spermatogonia, suggesting that PLZF not only plays an important role in the biological function of spermatogonial stem cells, but also is a convincing marker of undifferentiated spermatogonia. Several studies have applied PLZF as a marker to study progenitor germline cells $(19,20)$.

$\gamma \mathrm{H} 2 \mathrm{AX}$ has been widely recognized as a classical marker of DNA damage $(21,22)$. ATM promotes the phosphorylation of $\gamma \mathrm{H} 2 \mathrm{AX}$ at specific sites to form $\gamma \mathrm{H} 2 \mathrm{AX}$ after DNA DSBs (23). The $\gamma \mathrm{H} 2 \mathrm{AX}$ expression is helpful to evaluate the extent of DNA damage (24). IF staining of $\gamma \mathrm{H} 2 \mathrm{AX}$ showed that $\gamma \mathrm{H} 2 \mathrm{AX}$ was still expressed on the spermatogenic cells of the seminiferous tubules after lowdose IR, suggesting that the IR at a rational dose in this study still causes DSBs in the germline cells. Our previous studies revealed that the DNA repair response in the germline stem cells was a unique process independent of 
$\gamma \mathrm{H} 2 \mathrm{AX}(6)$. In the present study, results showed PLZF(+) spermatogonias were negative to $\gamma \mathrm{H} 2 \mathrm{AX}$, which is consistent with our previous results. Other studies imply that differentiated germ cells may undergo different repair mechanisms after IR (25). Studies have revealed that $\gamma \mathrm{H} 2 \mathrm{AX}$ can interact with P53 after IR to induce the apoptosis of differentiated spermatogonia, which is independent of DNA-PKcs (26). Moreover, Ku70, an important DNA damage repair protein, is also reported to be absent in early meiotic cells after formation of DNA breaks (27). However, our study revealed that both Ku70 and DNAPKcs were expressed on the undifferentiated spermatogonia while H2AX expression was absent, which suggests that the undifferentiated spermatogonias experience a unique mechanism after DSB. That is, there is a cell-tissue specific mechanism for the undifferentiated spermatogonia. Previous studies have reported that the DNA repair in the germline stem cells after IR was independent of $\gamma \mathrm{H} 2 \mathrm{AX}$, but it was a $53 \mathrm{bp} 1$ dependent process (28). Our results also confirmed that the undifferentiated spermatogonias were negative to $\gamma \mathrm{H} 2 \mathrm{AX}$ during the DNA damage repair, but the differentiated spermatogonias were positive to $\gamma \mathrm{H} 2 \mathrm{AX}$, which was consistent with previously reported. These findings confirmed that the mechanism of DNA repair after DSB in the undifferentiated spermatogonias is different from that in other cell types, suggesting a cellspecific mechanism of DNA repair in undifferentiated spermatogonias.

In present study, TUNEL was applied to detect the apoptotic cells in the testes. It indicated that low-dose IR also induced apoptosis of spermatogenic cells in the seminiferous tubules. By double staining of PLZF and TUNEL, the TUNEL positive cells were examined in the undifferentiated spermatogonia. Our results revealed that low-dose IR contributed to the apoptosis of undifferentiated germline cells which peaked at the acute stage of DNA damage in vivo.

In addition, low-dose IR also induced the expression of apoptosis-related proteins in the cultured undifferentiated spermatogonia. The expression of P53 and its active form p-P53 significantly increased after IR, which remained at $24 \mathrm{~h}$. Caspase- 3 protein expression was also up-regulated in the spermatogonia after IR, which continued to $24 \mathrm{~h}$ after IR. The expression of cleaved caspase- 3 was observed only in the acute phase ( 1 and $2 \mathrm{~h}$ ) of DNA damage post IR.

In order to further verify the apoptosis of undifferentiated spermatogonia in vitro, flow cytometry was done to detect the apoptosis of IR treated cells. Results showed apoptosis occurred immediately after IR, and continued to $24 \mathrm{~h}$.

It has been reported that low-dose IR may also cause DNA damage and induce DNA repair response in mammalian stem cells $(29,30)$. In the present study, lowdose cesium (Cs) irradiation was applied to establish cell and animal DNA damage models.

Although several experiments applied X-ray to induce DNA damage both in vivo and in vitro, both gamma-ray and beta-ray can be emitted by the ${ }^{137} \mathrm{Cs}$ irradiation resource, which is a major difference in the irradiation ray between ${ }^{137} \mathrm{Cs}$ and linear accelerator. Many studies have investigated the effects of IR on the murine testes, but the doses of X-ray used in available studies still remain high as compared to that in our study $(25,31)$.

As compared to the other studies about the effects of irradiation on the germline cells in vivo (32), low-dose IR was employed in our study, and results showed it could trigger the apoptosis of undifferentiated spermatogonia both in vivo and in vitro, which suggests that undifferentiated spermatogonias are more vulnerable and sensitive to DNA damage.

The in vivo and in vitro findings indicated that low-dose IR could induce the apoptosis of undifferentiated germline cells in vivo, which occurred mainly in the acute stage after IR, but the spermatogonia apoptosis mainly occurred at $24 \mathrm{~h}$ after IR in vitro. Moreover, caspase- 3 was involved in the process of apoptosis in the progenitor germline cells both in vivo and in vitro. The cleaved caspase- 3 was mainly expressed in the acute phase after IR, while flow cytometry indicated a large number of apoptotic cells at 24 h. Our findings suggested the germline cells apoptosis at different time points after IR, but more studies are needed to investigate the exact mechanism.

In summary, our results indicate that low-dose IR causes DNA damage to undifferentiated spermatogonia in vivo, and induce apoptosis of undifferentiated germline cells in a caspase- 3 dependent manner. Decreasing progenitor/germline stem cells are an important factor of male infertility. Thus, the apoptosis of undifferentiated germline cells is also crucial for the compromised development and quantity of sperms. Our results provide direct evidence on the low-dose IR induced apoptosis of mouse undifferentiated spermatogonia, which further demonstrate that the undifferentiated spermatogonias are sensitive to DNA damage. Our findings further suggest that the apoptosis of progenitor germline cells after IR changes over time. The apoptosis of undifferentiated spermatogonia occurs mostly at a longer time after IR 
in vitro as compared to that in vivo. The precise mechanism underlying the apoptosis of undifferentiated spermatogonia should be further elucidated. To date, few studies have been conducted to investigate the effects of low-dose IR on the germline stem cells. Our results provide evidence that IR causes apoptosis of undifferentiated spermatogonia cells and present a theoretical basis for the prevention and preservation of male infertility in the clinical radiation related treatments.

\section{Acknowledgments}

We gratefully appreciate Prof. Xiaoyu Liu, Ph.D. (Tongji University) for her useful suggestions. We also thank Prof. Xin Li, Ph.D. (University of Rochester) for providing PLZF antibody. English revision by Peipei Guo, Ph.D. (Weill Cornell Medical College) was also highly appreciated.

Funding: This study was supported by the Shanghai Natural Science Foundation (No. 19ZR1448700) and Shanghai Municipal Health and Family Planning Commission Project (No. 20174Y0067).

\section{Footnote}

Conflicts of Interest: The authors have no conflicts of interest to declare.

Ethical Statement: The authors are accountable for all aspects of the work in ensuring that questions related to the accuracy or integrity of any part of the work are appropriately investigated and resolved. The present study was approved by the Ethics Committee of Tongji Hospital (No. KYSB-2017-102).

\section{References}

1. Li W, Zeng Y, Zhao J, et al. Upregulation and nuclear translocation of testicular ghrelin protects differentiating spermatogonia from ionizing radiation injury. Cell Death Dis 2014;5:e1248.

2. Yasuda T, Oda S, Li Z, et al. Gamma-ray irradiation promotes premature meiosis of spontaneously differentiating testis-ova in the testis of p53-deficient medaka (Oryzias latipes). Cell Death Dis 2012;3:e395.

3. Liu G, Gong P, Zhao H, et al. Effect of low-level radiation on the death of male germ cells. Radiat Res 2006; 165:379-89.

4. Dohle GR. Male infertility in cancer patients: Review of the literature. Int J Urol 2010;17:327-31.

5. Meistrich ML. Effects of chemotherapy and radiotherapy on spermatogenesis in humans. Fertil Steril 2013;100:1180-6.

6. Le W, Qi L, Xu C, et al. Preliminary study of the homologous recombination repair pathway in mouse spermatogonial stem cells. Andrology 2018;6:488-97.

7. Aghajanyan A, Kuzmina N, Sipyagyna A, et al. Analysis of genomic instability in the offspring of fathers exposed to low doses of ionizing radiation. Environ Mol Mutagen 2011;52:538-46.

8. Tang FR, Loke WK. Molecular mechanisms of low dose ionizing radiation-induced hormesis, adaptive responses, radioresistance, bystander effects, and genomic instability. Int J Radiat Biol 2015;91:13-27.

9. Mohiuddin JJ, Baker BR, Chen RC. Radiotherapy for high-risk prostate cancer. Nat Rev Urol 2015;12:145-54.

10. Gunes S, Al-Sadaan M, Agarwal A, et al. Spermatogenesis, DNA damage and DNA repair mechanisms in male infertility. Reprod Biomed Online 2015;31:309-19.

11. Tegelenbosch RA, de Rooij DG. A quantitative study of spermatogonial multiplication and stem cell renewal in the C3H/101 F1 hybrid mouse. Mutat Res 1993;290:193-200.

12. Kubota H, Avarbock MR, Brinster RL. Spermatogonial stem cells share some, but not all, phenotypic and functional characteristics with other stem cells. Proc Natl Acad Sci U S A 2003;100:6487-92.

13. Kaucher AV, Oatley MJ, Oatley JM. NEUROG3 is a critical downstream effector for STAT3-regulated differentiation of mammalian stem and progenitor spermatogonia. Biol Reprod 2012;86:164, 1-11.

14. Niu Z, Goodyear SM, Rao S, et al. MicroRNA-21 regulates the self-renewal of mouse spermatogonial stem cells. Proc Natl Acad Sci U S A 2011;108:12740-5.

15. Kubota H, Avarbock MR, Brinster RL. Culture conditions and single growth factors affect fate determination of mouse spermatogonial stem cells. Biol Reprod 2004;71:722-31.

16. Liao HF, Chen WS, Chen YH, et al. DNMT3L promotes quiescence in postnatal spermatogonial progenitor cells. Development 2014;141:2402-13.

17. Lovelace DL, Gao Z, Mutoji K, et al. The regulatory repertoire of PLZF and SALL4 in undifferentiated spermatogonia. Development 2016;143:1893-906.

18. Buaas FW, Kirsh AL, Sharma M, et al. Plzf is required in adult male germ cells for stem cell self-renewal. Nat Genet 2004;36:647-52.

19. Costoya JA, Hobbs RM, Barna M, et al. Essential role of 
Plzf in maintenance of spermatogonial stem cells. Nat Genet 2004;36:653-9.

20. Grisanti L, Falciatori I, Grasso M, et al. Identification of spermatogonial stem cell subsets by morphological analysis and prospective isolation. Stem Cells 2009;27:3043-52.

21. Kuo LJ, Yang LX. Gamma-H2AX - a novel biomarker for DNA double-strand breaks. In Vivo 2008;22:305-9.

22. Siddiqui MS, Francois $M$, Fenech MF, et al. Persistent gammaH2AX: A promising molecular marker of DNA damage and aging. Mutat Res Rev Mutat Res 2015;766:1-19.

23. Baldock RA, Day M, Wilkinson OJ, et al. ATM Localization and Heterochromatin Repair Depend on Direct Interaction of the 53BP1-BRCT2 Domain with gammaH2AX. Cell Rep 2015;13:2081-9.

24. Lukas J, Lukas C, Bartek J. More than just a focus: The chromatin response to DNA damage and its role in genome integrity maintenance. Nat Cell Biol 2011;13:1161-9.

25. Ahmed EA, van der Vaart A, Barten A, et al. Differences in DNA double strand breaks repair in male germ cell types: lessons learned from a differential expression of $\mathrm{Mdc1}$ and 53BP1. DNA Repair (Amst) 2007;6:1243-54.

26. Hamer G, Roepers-Gajadien HL, van Duyn-Goedhart

Cite this article as: Qi L, Li J, Le W, Zhang J. Low-dose ionizing irradiation triggers apoptosis of undifferentiated spermatogonia in vivo and in vitro. Transl Androl Urol 2019;8(6):591-600. doi: 10.21037/tau.2019.10.16
A, et al. DNA double-strand breaks and gamma-H2AX signaling in the testis. Biol Reprod 2003;68:628-34.

27. Hamer G, Roepers-Gajadien HL, van Duyn-Goedhart A, et al. Function of DNA-protein kinase catalytic subunit during the early meiotic prophase without $\mathrm{Ku} 70$ and Ku86. Biol Reprod 2003;68:717-21.

28. Le W, Qi L, Li J, et al. Low-dose ionizing irradiation triggers a 53BP1 response to DNA double strand breaks in mouse spermatogonial stem cells. Syst Biol Reprod Med 2016;62:106-13.

29. Kalantari H, Motamed N, Mohseni Meybodi A, et al. The response of mouse embryonic stem cells to low doses of gamma-radiation: evidence for an adaptive response. Mutat Res Genet Toxicol Environ Mutagen 2014;761:44-7.

30. Manda K, Kavanagh JN, Buttler D, et al. Low dose effects of ionizing radiation on normal tissue stem cells. Mutat Res Rev Mutat Res 2014. [Epub ahead of print].

31. Beumer TL, Roepers-Gajadien HL, Gademan IS, et al. The role of the tumor suppressor p53 in spermatogenesis. Cell Death Differ 1998;5:669-77.

32. van der Meer Y, Huiskamp R, Davids JA, et al. The sensitivity to $\mathrm{X}$ rays of mouse spermatogonia that are committed to differentiate and of differentiating spermatogonia. Radiat Res 1992;130:296-302. 